\title{
LA CONCERTACIÓN DE LA EDUCACIÓN EN COLOMBIA
}

\author{
Francisco Cajiao $(*)$
}

SÍNTESIS: Los pactos sociales en la educación han tenido diferentes manifestaciones a lo largo del último siglo, dependiendo del contexto general del desarrollo del país y de las prioridades que el Estado ha ido definiendo con respecto a las necesidades de capital humano. El proceso de crecimiento demográfico, la urbanización acelerada y la presión ejercida sobre los gobiernos en relación con la ampliación de la cobertura en la educación básica, marcó un largo período en el cual el servicio educativo público fue asumido de manera muy centralizada por el gobierno, a la vez que la educación privada se expandió de forma notable, en especial en los principales centros urbanos del país. A partir de los noventa, la Asamblea Nacional Constituyente, influida por las corrientes internacionales y por las declaraciones de las grandes cumbres mundiales de educación, replanteó la participación social en el diseño de políticas públicas para los grandes temas nacionales, entre ellos la educación. Con este marco legal se llevaron a cabo dos grandes procesos de concertación nacional: el primero en torno a la Ley General de Educación, y el segundo para la formulación del primer Plan Decenal. Infortunadamente estas experiencias no alcanzaron a consolidarse, cuando ya se habían iniciado procesos de contrarreforma sin ninguna consulta pública.

SÍNTESE: Os pactos sociais na educação tiveram diferentes manifestações ao longo do último século, dependendo do contexto geral do desenvolvimento do país e das prioridades que o Estado tem defenindo com respeito às necessidades de capital humano. O processo de crescimento demográfico, a urbanização acelerada e a pressão exercida sobre os governos em relação à ampliação da cobertura na educação básica, marcou um longo período no qual o serviço educativo público foi assumido de maneira muito centralizada pelo governo, ao mesmo tempo em que a escola privada se expandiu de forma notável, especialmente nos principias centros urbanos do país. A partir dos anos noventa, a Assembléia Nacional Constituinte, influenciada pelas correntes internacionais e pelas declarações das grandes cúpulas mundiais de educação, repensou a participação social

(*) Consultor de las Naciones Unidas en El Salvador, Guatemala, Italia, Mozambique y Nicaragua; de la UNESCO en Perú, y de la CAF en los países del área andina. 
no desenho de políticas públicas para os grandes temas nacionais, entre eles a educação. Com este marco legal foram apresentados dois grandes processos de acordo nacional: o primeiro quanto à Lei Geral de Educação, e o segundo para a formulação do primeiro Plano Decenal. Desafortunadamente estas experiências não chegaram a consolidar-se, quando já haviam sido iniciados os processos de contra-reforma sem nenhuma consulta pública.

\section{INTRODUCCIÓN}

Para abordar las diferentes experiencias de concertación pública sobre el tema educativo, es muy importante tener una perspectiva general de la evolución del papel de la sociedad en relación con los procesos de incorporación social de las nuevas generaciones en los códigos culturales, que permiten que niños y jóvenes adquieran las herramientas necesarias para ser parte activa y productiva de sus comunidades.

Esta perspectiva histórica permite comprender la evolución de la percepción social de la educación, y el papel que juegan en diversos momentos las organizaciones ciudadanas y el Estado.

En la última década, a partir de la Constitución de 1991, Colombia ha buscado diversas alternativas para promover una amplia participación de la ciudadanía en la discusión y concertación de políticas públicas de educación, tal como lo han hecho otros países de América Latina. Las experiencias aún no han sido del todo decantadas, pero vale la pena reseñar lo que significaron dos de las más recientes y las perspectivas que existen sobre el tema.

En la evolución de la percepción de la educación a lo largo del siglo XX pueden distinguirse tres grandes momentos, que se relacionan con el proceso de modernización del país, y en los cuales los papeles de la comunidad y de la escuela tienen énfasis y características diferentes.

En un primer momento, que cubre más de la mitad del siglo, la educación es asumida como una tarea familiar y comunitaria, propia de las sociedades agrarias. Si bien en este período se produce un proceso activo de expansión de la escuela primaria, el nivel de escolarización es todavía muy precario y la permanencia de niños y niñas en el sistema se reduce a dos o tres años, sobre todo en el sector rural. En 1940 Colombia era todavía un país agrario, con más del $70 \%$ de su población situada en el sector rural. En 1945 sólo había 53 mil estudiantes matriculados en 
secundaria y 680 mil en primaria, mientras que más de la mitad de la población era analfabeta ${ }^{1}$. Los medios de comunicación eran fundamentalmente la prensa y la radio, ambas con una cobertura muy restringida, dadas las condiciones de analfabetismo y de deficiencia de la electrificación rural, y sólo en los años cincuenta se inicia la «revolución del transistor».

Bajo estas condiciones de desarrollo, el peso principal de la educación recae sobre la familia y sobre las comunidades adyacentes, que incorporan a niños y jóvenes a la cultura local y a la preparación para el trabajo en el contexto inmediato en el cual se definen los códigos de comunicación, los roles sociales y las destrezas necesarias para desempeñarlos.

En tal sentido, la educación no es cuestión de la escuela ni del Estado, sino del grupo familiar y de las incipientes organizaciones de las comunidades, que aseguran la continuidad y la supervivencia de sus miembros de una manera muy similar a la que prevaleció por milenios en los grupos humanos de todo el planeta.

En estos procesos de carácter antropológico existen unos importantes pactos sociales implícitos que provienen de la herencia cultural, permitiendo un gran nivel de coherencia y de conservación de las tradiciones mediante la codificación de ritos de iniciación y de paso, la delimitación de roles sexuales y de aprendizajes intergeneracionales, que abarcan los aspectos de la vida afectiva y reproductiva y los que se proyectan al mundo laboral. De esta forma, se configuran fuertes identidades locales en comunidades agrícolas, artesanales, pecuarias, etc. También se van definiendo modelos particulares de familia ${ }^{2}$, de intercambio comercial, de construcción de vivienda, de formas folclóricas y de celebraciones colectivas.

Los aprendizajes formales que introduce la escuela primaria desde el siglo XVIII se centran sobre todo en la adquisición de los códigos escritos literarios y matemáticos, y en la ampliación de la gama de información sobre geografía, historia nacional, catequesis, urbanidad y nociones muy generales de las ciencias naturales. Gradualmente, a lo

${ }^{1}$ Aline Helg (1987): La educación en Colombia, 1918-1957, Bogotá, CEREC.

${ }^{2}$ Cf. V. Gutiérrez de Pineda: Estructura, función y cambio de la familia en Colombia (2 vols.), Bogotá, Ascofame, 1975-1976. 
largo del siglo XX, las comunidades rurales aumentan su valoración por este tipo de educación, que ofrece a las nuevas generaciones una oportunidad que los mayores, casi todos analfabetos, no tuvieron. Es claro que lo que lleva la educación primaria al campo no pone en riesgo la identidad cultural de las comunidades, y, en cambio, ofrece una ventaja patente en el contexto de una sociedad que comienza a abrirse y a desplazarse hacia los centros urbanos.

De repente, a partir de 1950, el país comienza a dar saltos veloces en sus estructuras demográfica y productiva. Entre 1940 y 1965, la población total pasó de 8.600 .000 a un poco más de 17.000.000 de habitantes, transformándose del todo la estructura demográfica, pues en tanto la población rural creció en un 35\%, la urbana lo hizo en un 500\%. Junto con el acelerado proceso de urbanización, el país ingresó con rapidez a la red de comunicaciones modernas, primero con la introducción del radio-transistor, que entró con celeridad en el campo, y luego, a finales de los cincuenta, con la televisión, que en pocos años se difundió en los hogares de todos los niveles sociales. De forma simultánea con estos medios masivos, se incrementó la circulación de revistas cada vez mejor ilustradas y se ensancharon las redes de difusión de las telecomunicaciones: telefonía, distribución cinematográfica, publicidad visual en vallas, afiches y almanaques, etcétera.

Este proceso de transformación en la estructura demográfica dio paso a un segundo momento, en el cual la educación pasó a ser una prioridad en las políticas del Estado.

La creciente urbanización del país y la migración de campesinos a las ciudades plantea grandes problemas, pues esa gente llega la mayor parte de las veces buscando mejores oportunidades de supervivencia que en el campo, de modo que se trata de familias pobres, con muy poca capacidad de vincularse a la vida productiva de una sociedad que inicia su proceso de industrialización, con modelos laborales muy diferentes a los que conoce.

En este contexto, la escuela se convierte en la herramienta civilizadora por excelencia. Es imprescindible que niños y niñas adquieran las aptitudes necesarias para participar en la vida social y productiva que requiere la ciudad. Aunque el proceso civilizador de la escuela se inicia en el siglo XIX, la expansión escolar más fuerte, impulsada por el Estado, sólo se produce a mediados del siglo XX. Durante un largo período, que Ilega hasta la década de los 60 , el proceso escolar se centra en los 
cambios de hábitos de la población, en el que para este propósito cumplen un papel determinante los manuales de urbanidad.

A este respecto es muy interesante lo que plantea Patricia Londoño Vega en su trabajo "Cartillas y manuales de urbanidad y del buen tono, catecismos cívicos y prácticos para un amable vivir» ${ }^{3}$ :

Los cambios bruscos en el nivel de vida de una sociedad traen consigo el intento de imponer nuevos cánones de urbanidad y de comportamiento difundidos en cartillas, catecismos y manuales. Conocidos en Occidente desde el siglo XVI, proliferaron en el siglo XIX. En Colombia las clases altas, al incrementar su riqueza y al intensificarse el comercio con los países europeos hacia mediados del siglo XIX, se preocuparon por mejorar sus modales. Había bastante que aprender, pues con los nuevos consumos variaron utensilios de mesa y de cocina, modas, atuendos, muebles, elementos decorativos y hasta el sentido del gusto, alterando comportamientos y hábitos de vida. Se tradujeron varios manuales de urbanidad de Francia, referencia obligada del mundo "civilizado» en tales asuntos, y algunos autores locales, por lo regular ilustres personajes, redactaron otros inspirados en los europeos.

Los recetarios de modales hicieron parte del afán de civilización que hubo en el país, con particular fuerza en Antioquia, que desde el último cuarto del siglo antepasado fue a la cabeza en logros educativos, y que, al cambiar el siglo, encabezó la modernización económica. Medellín, que pasó de pueblo a centro fabril, atrajo trabajadores de sitios aledaños, a una oleada de ricos pueblerinos y a estudiantes enviados por sus familias a la Escuela de Minas o a la Universidad de Antioquia. Para ellos, por encima del origen social, el buen tono era una marca de la gente distinguida. Los modales, una manera de mejorar la posición social. Con razón afirma Carrasquilla en su novela Grandeza (1910): «Más valorada que la familia, aún más que el dinero, eran el buen porte, el buen gusto, el buen trato». Proliferaron manuales, cartillas, catecismos y códigos de urbanidad, de economía doméstica, de puericultura, higiene y temperancia, todos ampliamente divulgados. En esos años de orgullo y de optimismo, lo tradicional y lo campesino se tildaron de "cursis», «ñapangos» $y$ «mazamorrones». Un personaje de Carrasquilla, Ligia Cruz, recién Ilegada de Segovia, fue reprendida por llamar «madrina» a doña Emesia, pues "aquí no se usan esas familiaridades tan vulgares de los pueblos». La ansiedad de dar con el «buen tono» llevó a más de un

${ }^{3}$ Tomado de la Revista Credencial Historia (1997), Bogotá, edición 85, enero. 
pequeño rico pueblerino a acicalarse en demasía. Eso les pasó a Francisco de Paula Renden y a Tomás Carrasquilla cuando llegaron de Santodomingo a estudiar en la Universidad de Antioquia. Antonio José «Ñito» Restrepo recuerda que sus compañeros se burlaban de ellos por «pepitos», «cachacos» y «filipichines». Años más tarde, el periódico satírico Sancho Panza comentó: «No hay mayores metepatistas que algunos estudiantes de poblaciones, hijos de mayordomos acaudalados o cuando más de un boticario almacenista analfabeta. No han caído en esta ciudad y ya se creen unos señores meritísimos, muy chic e irresistibles».

El Estado, entonces, se ve frente a la necesidad de expandir las escuelas y las universidades para adecuarse a los nuevos retos planteados por la industrialización y el desarrollo de los sectores económicos modernos: banca, comercio a gran escala, servicios públicos... En Colombia, como en otros países similares, la educación se convierte en una prioridad, no sólo por su papel en el desarrollo, sino como forma de consolidar el sistema democrático. A pesar de que el orden político está montado sobre un fuerte centralismo presidencialista, es preciso contar con una clase dirigente, que, más allá de los grandes centros urbanos, pueda asumir las responsabilidades de gobierno en departamentos y municipios, para lo cual lo mínimo que se puede exigir es que la población tenga un nivel educativo básico.

De este modo, se pasa de un compromiso muy centrado en las familias y en las comunidades a un control casi exclusivo del Estado sobre el sistema escolar. Las obligaciones que emanan de la carta de los Derechos Humanos en torno a la educación suman nuevas presiones, para que los gobiernos hagan crecientes esfuerzos destinados a posibilitar el acceso de toda la población a los servicios educativos. Hasta la década de los 70 la oferta educativa crece de forma más o menos desordenada, en tanto que, sin mayor planificación, el gobierno central y los gobiernos departamentales se empeñan en la expansión de infraestructuras, en la designación de maestros y en la creación de universidades que puedan satisfacer la creciente demanda de la población. En este proceso, que culmina a mediados de los setenta con la Ley de Nacionalización de la Educación Pública, la participación de las comunidades se expresa en una presión constante sobre los gobernantes para que abran nuevas escuelas y colegios, pero lo que se hace en ellos queda bajo la potestad exclusiva del Estado. 
Eso lleva a un proceso relativamente rápido de modernización a partir de la década de los 60, introduciéndose metodologías, currículos actualizados y mecanismos de control y evaluación en los cuales participan diversas misiones extranjeras, y se comienza a formar una elite de expertos educativos nacionales formados en su mayor parte en el extranjero.

En los treinta años que transcurren entre 1950 y 1980, la percepción pública de la educación sufre cambios muy importantes, pasando de ser un recurso precioso de ascenso social o de conservación de privilegios -en esa dirección se explica el crecimiento de la educación privada en las zonas urbanas donde se concentran los grupos de poder político y económico-, a convertirse en un derecho fundamental útil como medio para el progreso económico y como vía fundamental para el desarrollo del individuo como sujeto social. El Estado, ahora, no es el proveedor de un servicio deseable para la buena marcha de la sociedad, sino el garante de un derecho fundamental e inalienable.

Además, la escuela comienza a verse con nuevas sensibilidades, en tanto que asegura el cuidado y el bienestar de niños y niñas. En efecto, el tema de la educación inicial adquiere nuevo sentido, y la posibilidad de acceso a la educación deja de tener significación si no está acompañada de estrategias que aseguren la permanencia y la calidad que se deben ofrecer a niños, niñas y jóvenes. De forma gradual surgen nuevas exigencias a las instituciones escolares, de las cuales se espera que sean semilleros eficaces de convivencia y de ciudadanía, y escenarios para tener contacto con la ciencia, la tecnología y el arte.

A lo largo de casi todo el siglo XX, la educación adquiere una inercia de crecimiento en la que la discusión pública prácticamente no existe. El sistema educativo reproduce de manera espontánea la fragmentación social, produciendo un modelo de educación pública a cargo del Estado para los más pobres y para las comunidades más aisladas, mientras que prolifera un sistema paralelo de educación privada que aún hoy representa un porcentaje muy elevado de la matrícula, sobre todo en las capitales donde se concentran los polos de desarrollo. Para la población con mayores recursos se establece como pauta el bachillerato clásico conducente a la universidad, en tanto la educación técnica, que prepara para el mercado laboral, se identifica con una educación para jóvenes de bajos recursos que es muy probable que no lleguen a tener la oportunidad de cursar niveles avanzados de formación profesional. 
Esta forma de configurarse la educación no es objeto de debate público ni de análisis en los medios de comunicación. Las noticias que aparecen en la prensa se refieren más que nada a situaciones coyunturales relacionadas con el conflicto permanente entre los maestros y el gobierno, a situaciones de crisis en las universidades, o a reportar logros concretos de los gobiernos. En efecto, la centralización de los temas educativos en la mente de los ministros del ramo hace que la discusión de los grandes temas del sector queden reducidos a las continuas confrontaciones y negociaciones entre el gobierno y las asociaciones sindicales del magisterio oficial, en las que priman asuntos de carácter reivindicativo de diversa índole: salarios, compensaciones, calendarios, horas de trabajo, prestaciones, etc. Por otra parte, y de forma paralela, se producen las mismas concertaciones y los mismos conflictos con las asociaciones de colegios privados por asuntos que se refieren a control de matrículas y a otras reglamentaciones relativas a la creación y aprobación de nuevos planteles. Con las universidades ocurre otro tanto, dependiendo de los intereses específicos de las públicas y de las privadas.

Todo esto genera un modelo de desarrollo de las políticas como resultado de una concertación privada entre grupos de interés y el Estado, en el que la opinión ciudadana no cuenta. Ni los empresarios, ni las familias, ni las organizaciones de la sociedad civil intervienen en las grandes orientaciones. En el Congreso se crea la legislación que responde a los acuerdos pactados por el ministro de educación para impulsar las reformas previamente convenidas, y luego, en las dependencias especializadas, se llevan a cabo los desarrollos reglamentarios, generándose una telaraña legal casi indescifrable. Por su parte, dentro del gobierno se negocian las asignaciones presupuestales, dependiendo de la capacidad del ministro de turno de influir en las decisiones, que, con frecuencia, son tomadas más para evitar conflictos de alto costo político para el gobierno, que para asegurar planes a largo plazo.

Es muy posible que estas características de la evolución de las políticas educativas colombianas sean similares a las de otros países en lo que se refiere a su condición de coyunturales y de corto plazo, y en la casi total ausencia de un debate público que pueda hacer confluir los intereses generales en la definición de un horizonte coherente resultante de grandes pactos sociales.

El tercer momento en la evolución de la percepción social de la educación se materializa con la Constitución Política de 1991, influida en gran parte por el contexto internacional. 
En 1989 la Asamblea General de las Naciones Unidas adoptó por unanimidad la Convención sobre los Derechos del Niño, cuyas disposiciones, aplicables a la población mundial menor de 18 años, se refieren a cuatro grandes temas: supervivencia, desarrollo, protección y participación. Entre las normas adoptadas se incluye de forma clara la educación como un derecho de los niños. A partir de este instrumento jurídico internacional, se desarrollaron la Conferencia Mundial de Educación para Todos, en Jomtien (1990), que aprobó la Declaración Mundial de Educación para Todos y el Marco de Acción para satisfacer las necesidades básicas de aprendizaje; la Cumbre Mundial a favor de la Infancia, en Nueva York (1990); y el Plan de Acción de la UNESCO para la erradicación del analfabetismo. Antes se había realizado, a nivel regional, el Proyecto Principal de Educación para América Latina y el Caribe (OREALC-UNESCO) en Quito (1981).

En los textos de las declaraciones mencionadas aparecen numerosas referencias a la necesidad urgente de cambiar la percepción de la educación por parte de los Estados y de la sociedad en su conjunto. En la Declaración Mundial de Educación para Todos hay tres objetivos que tienden a la construcción de pactos sociales amplios:

- Promover una visión ampliada de la educación básica, que vaya más allá de los recursos actuales, de las estructuras institucionales, de los planes de estudio y de los sistemas tradicionales de instrucción.

- Fortalecer la concertación de acciones.

- Movilizar todos los recursos financieros y humanos, públicos, privados o voluntarios.

El conjunto de orientaciones resultantes de las cumbres internacionales dio origen en Colombia a numerosos grupos de trabajo, que participaron activamente en la reparación de las discusiones que se llevaron a cabo después en la Asamblea Constituyente de 1991. Abel Rodríguez, que formó parte de la Asamblea, indica «Que [aunque] la educación no haya sido objeto de intensas controversias ni un asunto prioritario en la agenda del proceso constituyente, no quiere decir que no haya merecido una atención justa y debida. Durante el período de preparación de la Asamblea, un grupo de científicos, artistas, educadores y trabajadores de la cultura integró una subcomisión adscrita a la Comisión de Derechos Humanos, que trabajó sobre la temática "Derecho a la educación, fomento a la cultura, la ciencia y la tecnología". De esta 
subcomisión, un grupo numeroso se ocupó en forma exclusiva del tema educativo. Las comisiones y subcomisiones preparatorias de la Asamblea Nacional Constituyente fueron organizadas por el gobierno para facilitar la participación de voceros representativos de la opinión en el debate y en la formulación de propuestas de reforma constitucional. En total, funcionaron diez comisiones que se distribuyeron en veintinueve subcomisiones» ${ }^{4}$.

En la Asamblea, cuatro de las cinco comisiones de trabajo se ocuparon de la educación: la primera la examinó como un derecho, la segunda como competencia de las entidades territoriales, la tercera como responsabilidad de los órganos nacionales del Estado, y la quinta como asunto de la economía y de la hacienda pública. De los 380 artículos que integran el texto constitucional, cerca de 40 se ocupan total o parcialmente de la educación y de la cultura. A través de la normativa, la educación se asume como elemento esencial de la dignidad humana y como una actividad inherente a la finalidad social del Estado.

La Asamblea Nacional Constituyente, en sí misma, constituye un enorme esfuerzo de confluencia del país hacia un nuevo pacto social, en medio de una profunda crisis social y política. Abel Rodríguez continúa: «A pesar de que la educación constituía uno de los problemas sociales más agudos, dada la crisis en la que la habían sumido las políticas públicas aplicadas durante la década de los ochenta, y el interés de diversos sectores por convertirla en un asunto de prioridad nacional, ésta no alcanzó a figurar entre las grandes preocupaciones que motivaron el proceso constituyente del 91 . Otros problemas más agobiantes, como la violencia, el terrorismo, la corrupción, el clientelismo, etc., copaban los pocos espacios públicos de discusión que existían por ese entonces».

Pero al plantear una nueva concepción de la participación democrática, otro modelo de organización del Estado y un régimen de descentralización que antes no existía, la Constitución se convirtió en un instrumento pedagógico que impulsó nuevas formas de hacer las cosas, invitando a la ciudadanía a ocuparse de temas que antes eran un patrimonio casi exclusivo del poder ejecutivo.

${ }^{4}$ A. Rodríguez (2002): La educación después de la Constitución del 91, Bogotá, Magisterio, p. 27. 


\section{LA LEY GENERAL DE EDUCACIÓN}

En el caso específico de la educación, el primer esfuerzo importante de acercamiento a lo que podríamos Ilamar un pacto social por la educación fue el proceso de elaboración de la Ley General de Educación, que se aprobó en 1994, después de un amplio proceso de discusión y de consultas.

Su origen provino de la iniciativa de FECODE de concertar con el gobierno una ley que regulara de manera general la educación nacional. Esta iniciativa fue muy bien recibida por la comunidad educativa y por otros sectores del país, pues respondía a un clima de discusión y participación que se había desencadenado con el proceso constituyente. Era tan propicio el ambiente de concertación, que el propio Ministro de Educación, Carlos Holmes Trujillo, superó la iniciativa del magisterio proponiendo una "Constituyente Educativa».

Sin embargo, el proceso dio lugar a fuertes controversias y debates entre las concepciones más democráticas y las conservadoras. Al respecto, A. Rodríguez señala que «la iniciativa de la Constituyente Educativa, que tuvo una amplia acogida por parte de diversas organizaciones y grupos académicos independientes, fue objetada por el Comité Ejecutivo de FECODE [...]. El debate librado alrededor de este asunto se dirimió a favor de los sectores gremiales, encabezados por la dirección de FECODE, y el gobierno, que, aliados, impusieron el procedimiento de la negociación bilateral. De esta manera, el proceso arrancó desconociendo el principio que postula que la educación es un asunto de todos» ${ }^{5}$.

Un segundo aspecto de debate enfrentó a quienes defendían la necesidad de una ley estatutaria que regulara el derecho a la educación como derecho fundamental, y un sector que se inclinaba por una ley que reglamentara la prestación del servicio educativo. En este último grupo se inscribió FECODE, sosteniendo que en la Constitución no estaba explícita la definición de la educación como derecho fundamental, a pesar de que las sentencias de la Corte Constitucional ratificaban el carácter fundamental del derecho a la educación. Al perderse la oportunidad de avanzar en la dirección indicada por la Corte Constitucional, la necesidad de regulación del derecho a la educación quedó pendiente, y, por ahora, no hay indicios de que se vaya a avanzar en esa tarea legislativa.

${ }^{5}$ A. Rodríguez, op. cit., p. 120. 
El tercer motivo de controversia se produjo en torno al contenido y a los alcances de la ley, que al principio sólo tuvo lugar entre el gobierno y el Comité Ejecutivo de FECODE, siguiendo los proyectos intercambiados para adelantar la discusión. En un segundo momento el debate se centró en el proyecto de ley acordado entre los mismos actores para presentarlo al Congreso.

Sin duda, el proyecto de ley acordado entre el gobierno y los educadores significó importantes reformas para el progreso de la educación, pero muchas de ellas fueron criticadas con dureza por grupos académicos y gremiales excluidos de la discusión, pues era evidente que se estaba perdiendo una valiosa oportunidad para hacer reformas más profundas y de mayor perspectiva en la transformación del sistema educativo. A pesar de todo, no hay que negar la importancia que tuvo el hecho de que, por primera vez, un proyecto de reforma educativa llegara al Congreso con el apoyo del gobierno y de las organizaciones magisteriales.

A partir de la radicación del proyecto de ley en la Cámara de Representantes (Diputados), los integrantes de la Comisión Sexta Constitucional y los ponentes de la ley convocaron una serie de foros en distintas ciudades del país, que sirvieron para expresar diferentes puntos de vista sobre el contenido y los alcances del proyecto. Muchas de las sugerencias generadas en los foros fueron desatendidas por los parlamentarios, pero resultó evidente que se creó un clima de reflexión favorable al proceso de trámite de la ley, que hasta entonces era desconocido para el sector educativo, acostumbrado de siempre a recibir las leyes y normas como sorpresas cocinadas en la privacidad de los despachos ministeriales.

Al llegar al Senado, el proyecto de ley tomó un nuevo rumbo, pues los ponentes designados por la corporación no acudieron a foros sino a consultas directas, sobre todo con voceros de instituciones educativas privadas y con expertos en educación provenientes de diversas organizaciones, a fin de recoger la opinión ciudadana sobre el citado proyecto. Después de un laborioso proceso la ponencia fue aceptada, y estuvo acompañada de un largo pliego que modificaba en gran parte el proyecto aprobado por la Cámara de Representantes. Finalmente, la Ley 115 de 1994 fue promulgada, acogiendo la mayoría de las modificaciones propuestas por los ponentes.

Aunque el tema central del presente artículo no es el contenido de esta ley sino el proceso que condujo a su preparación y promulgación, 
es bueno señalar que representa un notable avance sobre las normas que rigieron la educación colombiana durante el siglo XX, pues a pesar de las discrepancias surgidas en el proceso, introdujo un conjunto de reformas con enorme capacidad de hacer cambios importantes en la educación a mediano y a largo plazo. Muchos temas que preocupaban a los educadores y al conjunto de la sociedad fueron incluidos en la ley, que fijó los fines del sistema educativo, estableció las modalidades de atención a poblaciones especiales y a grupos étnicos promoviendo su integración al sistema de educación regular, fortaleció la autonomía de las instituciones educativas, creó órganos de participación en todos los niveles, impuso al Ministerio de Educación la obligación de elaborar planes decenales, y creó mecanismos de participación en la formulación de políticas para la sociedad civil, como las juntas y los foros educativos.

A pesar de las virtudes de la ley quedaron vacíos y limitaciones, como dejar fuera de la estructura del sistema a la educación superior, y omitir regular el carácter público del servicio educativo consagrado en la Constitución.

Sopesando virtudes y carencias, puede afirmarse que los avances logrados tienen mucha mayor relevancia que las limitaciones. Y, más allá del articulado de la ley, fue de trascendental importancia la participación de amplios sectores del magisterio en el proceso de discusión previo, pues sentó un precedente en la apropiación pública del tema educativo. Es verdad que la idea original de una "Constituyente Educativa» representaba mejor el espíritu de participación ciudadana que se había generado con la Asamblea Constituyente, porque bajo esa figura se hubiera podido incluir de manera más activa al sector universitario, a los empresarios y a otros sectores del propio gobierno.

Pero la participación del magisterio en los debates que condujeron a la promulgación de la ley tuvo su impacto en el contenido y en los alcances de la norma, y generó un compromiso con muchos de los temas definidos como orientación del sistema, pues al haber sido parte de un pacto social, era ineludible que se creara una obligación sobre los contenidos que hicieron parte del debate.

Infortunadamente no puede decirse lo mismo del gobierno, que para el caso de la Ley General de Educación estaba en la mente casi exclusiva del ministro de Educación, que mientras se avanzaba en el proceso de discusión de la reforma educativa, presentó de manera unilateral el proyecto de ley sobre distribución de competencias y 
recursos que se convirtió en la Ley 60 de 1993. Esta ley, aprobada antes que la 115 , entraba en conflicto con algunos temas cruciales de la administración que se habían pactado con FECODE en el marco de la Ley de Educación, lo cual dio lugar a un paro indefinido de las actividades docentes.

\section{EL PLAN DECENAL DE EDUCACIÓN}

En 1996 se expidió el Plan Decenal de Educación 1996-2005, de acuerdo con la disposición contenida en la Ley 115 de 1994, que establece que «El Ministerio de Educación Nacional, en coordinación con las entidades territoriales, preparará por lo menos cada diez años el Plan de Desarrollo Educativo, que incluirá las acciones correspondientes para dar cumplimiento a los mandatos constitucionales y legales sobre prestación del servicio educativo. Este plan tendrá carácter indicativo, será evaluado, revisado permanentemente y considerado en los planes nacionales y territoriales de desarrollo».

Conviene citar lo que señala Abel Rodríguez, quien coordinó desde el Ministerio de Educación una parte del proceso de seguimiento inicial de este proceso, para comprender los alcances del mecanismo establecido en la ley para promover la participación de la ciudadanía en la construcción de pactos sociales a largo plazo:

«El Plan Decenal fue concebido para cumplir una doble función: de un lado, servir como instrumento de planeación del desarrollo educativo a nivel nacional, departamental, distrital, municipal e institucional; y, del otro, servir como instrumento de movilización de la opinión pública y de la ciudadanía por la educación.

La misión del Plan Decenal es darle continuidad y visión de futuro a las políticas educativas. Su utilidad práctica es servir de carta de navegación para realizar las transformaciones educativas que el país requiere. Visto en esta perspectiva, el Plan 1996-2005 señaló el horizonte hacia donde debía dirigirse la educación en el decenio.

Al igual que su elaboración, la ejecución de un Plan Decenal pasa por la realización de una gran movilización nacional de opinión, fundamentada en la comprensión de que la educación es un asunto de 
orden estratégico que involucra el interés de toda la comunidad nacional» 6 .

La elaboración del Plan fue una iniciativa de diversas organizaciones de la sociedad civil, entre las cuales había fundaciones, corporaciones, centros de investigación y movimientos cívicos, y encontró muy buena acogida por parte del presidente de la República, Ernesto Samper, y de su ministra de Educación, María Emma Mejía, quienes expidieron el decreto 1719 de octubre de 1995, dando pautas para la preparación y formulación del Plan.

Para la elaboración del Plan se recurrió a diversos mecanismos de participación, entre los cuales se destacaron las mesas de trabajo (cerca de 150 en todo el país); los encuentros de secretarios de educación, de universidades y de centros de investigación; los foros (se Ilevaron a cabo dos nacionales y cerca de 300 regionales y locales); los pronunciamientos públicos; la elaboración y publicación de documentos; teleconferencias; y comisiones técnicas. Dentro de este proceso, es necesario destacar el protagonismo del Ministerio de Educación como animador, y la participación de las entidades territoriales a través de las secretarías de educación. También fue de gran importancia la participación de ONG y de los medios de comunicación.

Desafortunadamente no puede decirse lo mismo de la comunidad académica, del sector productivo y de los sindicatos, que se mantuvieron al margen del proceso.

El Plan, que no estuvo exento de predominio de lo político sobre lo técnico, y que fue escenario de importantes debates sobre la pertinencia de la educación en el contexto de un proyecto de país; la presencia de lo regional y de lo local; la relación entre educación y desarrollo económico y el papel definitivo de las instituciones escolares, fue finalmente aprobado por el presidente de la República y por la ministra de Educación Nacional el 23 de febrero de 1996.

Señala Abel Rodríguez que, al repasar las normas constitucionales y las leyes que la desarrollan en materia educativa, se encontrará que el Plan Decenal es el que mejor se ajusta a sus contenidos y más se aproxima a su realización. Por desgracia, antes que el Plan ejecutara los mandatos que buscaba cumplir, comenzaron a reformarse.

${ }^{6}$ A. Rodríguez, op. cit., p. 173. 
En efecto, terminado el mandato del presidente Samper, se pudo comprobar que en las altas esferas del poder no había existido una clara comprensión de lo que significó el proceso de elaboración de un plan de este alcance. En el mejor de los casos, se elogió la movilización social conseguida por quienes alentaron el desarrollo de la discusión pública, pero se ignoraron los contenidos y las orientaciones que se habían logrado con la participación ciudadana. Tan fue así, que el siguiente gobierno ni siquiera consideró el Plan Decenal como orientador del Plan Nacional de Desarrollo 1998-2000, tal como dispone el artículo 72 de la Ley 115 de 1994.

Si para los gobiernos siguientes (el de Andrés Pastrana y el actual de Álvaro Uribe) el Plan fue letra muerta, puede decirse algo similar de las organizaciones de la sociedad civil y de la prensa, que tampoco encontraron interés o instrumentos para presionar al gobierno a que tomara en cuenta el resultado de la concertación pública.

A pesar de la obligación legal de revisar el Plan y de elaborar por lo menos uno cada diez años, el actual gobierno no ha mencionado nada en relación con la estrategia que debería seguirse para el próximo, dado que el actual se hizo con el horizonte del año 2005.

Con independencia de la precaria efectividad del primer Plan Decenal, de la cual han sido responsables los dos últimos gobiernos, de corte tecnocrático y fieles seguidores de las políticas neoliberales que se han impulsado en la educación de casi todo el continente, este continúa siendo la mejor y más válida estrategia para dotar al país de una política de Estado en el campo de la educación, concertada a escala nacional.

En los últimos ocho años, la política educativa ha vuelto a centrarse en planes y programas cocinados en el Ministerio de Educación, con énfasis en el desarrollo de mecanismos de control y de estrategias de eficiencia, dejando de lado las grandes discusiones sobre el sentido del Sistema Educativo en relación con el desarrollo humano y con las grandes problemáticas del país. A esta tendencia de corte más tecnocrático han contribuido la profunda crisis económica que se evidenció a partir de 1998, el fracaso del proceso de negociación con los grupos armados, y el impulso de la estrategia de seguridad democrática que aumenta de forma notoria el presupuesto militar. Así, la participación ciudadana en la discusión de la educación ha dejado de ser prioritaria. 
De cualquier manera, las experiencias de amplia participación ciudadana demostraron que sí era posible un nivel de discusión civilizada que concluyera con resultados positivos de concertación, que generaron nuevos espacios de discusión que se mantienen en todo el país, y que, de una u otra forma, alimentan nuevas experiencias y procesos locales y departamentales. En algunas entidades territoriales se han elaborado planes decenales y en otras están en proceso de discusión.

Es evidente que una tradición de siglos de silencio no se transforma en una década de enunciados democráticos.

\section{BIBLIOGRAFÍA} Magisterio

CAJIAO, F. (2003): La formación de maestros y su impacto social, Bogotá,

HeLG, A. (1987): La Educación en Colombia, 1918-1957, Bogotá, CEREC.

LONDOÑo, P. (1997): «Cartillas y manuales de urbanidad y del buen tono, catecismos cívicos y prácticos para un amable vivir», Bogotá, Revista Credencial Historia, edición 85, enero.

MINISTERIO DE EDUCACIÓN NACIONAL (1996): Plan decenal de educación (19962005), Colombia, http://www.mineducación.gov.co/plan-estratégico. Magisterio.

RodRíGUEZ, A.(2002): La educación después de la Constitución del 91, Bogotá,

SALDARRIAGA, O. (2003): Del oficio de maestro, Bogotá, Magisterio.

\section{Leyes}

- Constitución Política de Colombia (1991).

- LEY 115 DE 1994.

- LEY 60 DE 1993. 


\title{
Contactar
}

Revista lberoamericana de Educación

\author{
Principal OEI
}

